\title{
GENERALISATTONS OF SOME HYPERGEOMETRIC FUNCTION TRANSFORMATIONS
}

\author{
by T. M. MACROBERT \\ (Received 3rd January, 1949)
}

§ 1. Introductory. The formulae

$$
F\left(\begin{array}{c}
\alpha, \beta ; x \\
\gamma
\end{array}\right)=F\left\{\begin{array}{c}
\frac{1}{2} \alpha, \frac{1}{2} \beta ; 4 x(1-x) \\
\gamma
\end{array}\right\},
$$

where $\gamma=\frac{1}{2}(\alpha+\beta+1)$, and

$$
F\left(\begin{array}{c}
\alpha, \beta ; x \\
\gamma
\end{array}\right)=(1-x)^{-\alpha} F\left\{\begin{array}{c}
\frac{1}{2} \alpha, \frac{1}{2}+\frac{1}{2} \alpha-\beta ; \\
\gamma
\end{array} \frac{-4 x}{(1-x)^{2}}\right\},
$$

where $\gamma=1+\alpha-\beta$, were given by Gauss (Ges. Werke, iii, pp. 225, 226). It is here proposed to find the corresponding expressions for the hypergeometric function when $\gamma$ has general values (not zero or negative integral). These will be derived in section 2 by applying Lagrange's expansion

$$
F(x)=F(\lambda)+\sum_{n=1}^{\infty} \frac{w^{n}}{n !} \frac{d^{n-1}}{d \lambda^{n-1}}\left[F^{\prime}(\lambda)\{\phi(\lambda)\}^{n}\right],
$$

where

$$
x=\lambda+w \phi(x)
$$

and that root of equation (4) in $x$ is taken which is equal to $\lambda$ when $w=0$. Two generalisations of Whipple's Transformation will be obtained in section 3 .

§ 2. Expressions for the Hypergeometric Function. Let $x=\frac{1}{2}\{1-\sqrt{ }(1-\xi)\}$, so that

$$
x=\frac{1}{4} \xi(1-x)^{-1}
$$

and in Lagrange's Expansion put $\lambda=0, w=\frac{1}{4} \xi=x(1-x)$ and $\phi(x)=(1-x)^{-1}$. Then

$$
\begin{aligned}
& F\left(\begin{array}{c}
\alpha, \beta ; x \\
\gamma
\end{array}\right)=1+\sum_{n=1}^{\infty} \frac{w^{n}}{n !}\left[\frac{d^{n-1}}{d \lambda^{n-1}}\left\{F^{\prime}\left(\begin{array}{c}
\alpha, \beta ; \lambda \\
\gamma
\end{array}\right)(1-\lambda)^{-n}\right\}\right]_{\lambda=0} \\
& \quad=1+\sum_{n=1}^{\infty} \frac{w^{n}}{n !}\left\{\frac{(\alpha ; n)(\beta ; n)}{(\gamma ; n)}+{ }^{n-1} C_{1} \frac{(\alpha ; n-1)(\beta ; n-1)}{(\gamma ; n-1)}(n ; 1)+\ldots\right\},
\end{aligned}
$$

where $(\alpha ; 0)=1$ and $(\alpha ; n)=\alpha(\alpha+1) \ldots(\alpha+n-1)$.

Thus

$$
F\left(\begin{array}{c}
\alpha, \beta ; x \\
\gamma
\end{array}\right)=\sum_{n=0}^{\infty}\{x(1-x)\}^{n} \frac{(\alpha ; n)(\beta ; n)}{n !(\gamma ; n)} F\left(\begin{array}{l}
1-n, n, 1-\gamma-n ; 1 \\
1-\alpha-n, 1-\beta-n
\end{array}\right) .
$$

This formula is the generalisation of (1). If the order of the terms in the generalised hypergeometric series on the right is reversed the expansion may be written

where

$$
F\left(\begin{array}{c}
\alpha, \beta ; x \\
\gamma
\end{array}\right)=1+\frac{\alpha \cdot \beta}{\gamma} \sum_{n=1}^{\infty}\{x(1-x)\}^{n} \frac{(2 n-2) !}{n !(n-1) !} \psi(n ; \alpha, \beta ; \gamma),
$$

$$
\psi(n ; \alpha, \beta ; \gamma)=\text { first } n \text { terms of } F\left(\begin{array}{c}
\alpha+1, \beta+1,1-n ; 1 \\
\gamma+1,2-2 n
\end{array}\right) \text {. }
$$


In considering the convergence of the series on the right, it is convenient to use form (6). Then

$$
\begin{aligned}
|\psi(n ; \alpha, \beta ; \gamma)| & \leqq \sum_{r=0}^{n-1}\left|\frac{(\alpha+1 ; r)(\beta+1 ; r)}{r !(\gamma+1 ; r)}\right| \frac{1}{2^{r}} \\
& <\sum_{r=0}^{\infty}\left|\frac{(\alpha+1 ; r)(\beta+1 ; r)}{r !(\gamma+1 ; r)}\right| \frac{1}{2^{r}}=K,
\end{aligned}
$$

where $K$ is a positive number independent of $n$. Hence the modulus of the $n$th term in the series on the right of $(6)$ is less than

$$
|4 x(1-x)|^{n} \frac{\left(\frac{1}{2} ; n-1\right)}{n !} K
$$

and, consequently, by the comparison and ratio tests, the series converges absolutely if

$$
|4 x(1-x)|<1 \text {. }
$$

Conversely, let $\xi=\frac{1}{2}\{1-\sqrt{ }(1-x)\}$, so that $x=4 \xi \cdot \frac{1}{2}\{1+\sqrt{ }(1-x)\}=4 \xi(1-\xi) ;$ and, in Lagrange's Expansion, put $\lambda=0, w=4 \xi, \phi(x)=\frac{1}{2}\{1+\sqrt{ }(1-x)\}$; then

$$
F\left(\begin{array}{c}
\alpha, \beta ; x \\
\gamma
\end{array}\right)=1+\sum_{n=1}^{\infty} \frac{(4 \xi)^{n}}{n !}\left[\frac{d^{n-1}}{d \lambda^{n-1}}\left\{F^{\prime}\left(\begin{array}{c}
\alpha, \beta ; \lambda \\
\gamma
\end{array}\right)\left(\frac{1+\sqrt{ }(1-\lambda)}{2}\right)^{n}\right\}\right]_{\lambda=0} .
$$

But [Phil. Mag., Ser. 7, xxvI, p. 86], if $\alpha$ is not a positive integer,

$$
\left\{\frac{1+\sqrt{ }(1-\lambda)}{2}\right\}^{\alpha}=F\left(\begin{array}{c}
-\frac{1}{2} \alpha, \frac{1}{2}-\frac{1}{2} \alpha ; \lambda \\
1-\alpha
\end{array}\right)
$$

Hence, when $\alpha \rightarrow n$, a positive integer,

$$
\left\{\frac{1+\sqrt{ }(1-\lambda)}{2}\right\}^{n}=\text { the first } \frac{1}{2} n+\frac{1}{2} \text { or } \frac{1}{2} n+1 \text { terms of } F\left(\begin{array}{c}
-\frac{1}{2} n, \frac{1}{2}-\frac{1}{2} n ; \lambda \\
1-n
\end{array}\right)
$$

Therefore,

+ terms of degree not less than $n$ in $\lambda$.

$$
F\left(\begin{array}{c}
\alpha, \beta ; x \\
\gamma
\end{array}\right)=\sum_{n=0}^{\infty}[2\{1-\sqrt{ }(1-x)\}]^{n} \frac{(\alpha ; n)(\beta ; n)}{n !(\gamma ; n)} F\left(\begin{array}{c}
-\frac{1}{2} n, \frac{1}{2}-\frac{1}{2} n, 1-\gamma-n ; 1 \\
1-\alpha-n, 1-\beta-n
\end{array}\right) \text {. }
$$

On interchanging $x$ and $\xi$, it is seen that

$$
F\left\{\begin{array}{c}
\alpha, \beta ; 4 x(1-x) \\
\gamma
\end{array}\right\}=\sum_{n=0}^{\infty}(4 x)^{n} \frac{(\alpha ; n)(\beta ; n)}{n !(\gamma ; n)} F\left(\begin{array}{c}
-\frac{1}{2} n, \frac{1}{2}-\frac{1}{2} n, 1-\gamma-n ; 1 \\
1-\alpha-n, 1-\beta-n
\end{array}\right) .
$$

The series on the right is convergent if $|x|<\frac{1}{2}$ [Cf. previous paper].

Again, in the formula

$$
F\left(\begin{array}{c}
\alpha, \beta ; x \\
\gamma
\end{array}\right)=(1-x)^{-\alpha} F\left(\begin{array}{c}
\alpha, \gamma-\beta ; \\
\gamma
\end{array} \frac{x}{x-1}\right)
$$

apply (5) to the hypergeometric function on the right and get

$$
F\left(\begin{array}{c}
\alpha, \beta ; x \\
\gamma
\end{array}\right)=(1-x)^{-\alpha} \sum_{n=0}^{\infty}\left\{\frac{-x}{(1-x)^{2}}\right\}^{n} \frac{(\alpha ; n)(\gamma-\beta ; n)}{n !(\gamma ; n)} F\left(\begin{array}{c}
1-n, n, 1-\gamma-n ; 1 \\
1-\alpha-n, 1-\gamma+\beta-n
\end{array}\right) .
$$

This series, which is convergent for $x$ small and $\left|4 x /(1-x)^{2}\right|<1$, is the generalisation of (2).

Note.-Formulae (1) and (2) may be deduced from (5), (8) and (9) by using Saalschutz's iheorem or Whipple's theorem (W. N. Bailey, Generalized Hypergeometric Series, p. 16). 

written

§3. Generalisations of Whipple's Transformation. Whipple's Transformation may be

Now, if $x$ is small,

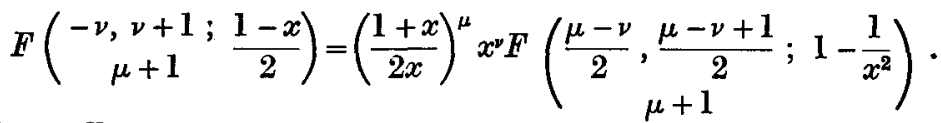

$$
(1-x)^{-1} \alpha \sum_{n=0}^{\infty}\left(\frac{x}{x-1}\right)^{n} \frac{(\alpha ; n)(\beta ; n)}{n !(\gamma ; n) 4^{n}} F\left(\begin{array}{l}
1-n, n, 1-\gamma-n ; 1 \\
1-\alpha-n, 1-\beta-n
\end{array}\right)=\sum_{n=0}^{\infty} \phi(n ; \alpha, \beta ; \gamma) x^{n}, \ldots
$$

where

$$
\begin{aligned}
& \phi(n ; \alpha, \beta ; \gamma) \\
& \quad=\sum_{r=0}^{n} \frac{\left(\frac{1}{2} \alpha+n-r ; r\right)(\alpha ; n-r)(\beta ; n-r)}{r !(n-r) !(\gamma ; n-r)(-4)^{n-r}} F\left(\begin{array}{c}
r+1-n, n-r, r+1-\gamma-n ; 1 \\
r+1-\alpha-n, r+1-\beta-n
\end{array}\right) .
\end{aligned}
$$

This formula can be established by expanding the powers of $(1-x)$ in $(11)$ in powers of $x$ and picking out the coefficient of $x^{n}$. Hence, on replacing $x$ by $x /(x-1)$, we have

$$
\stackrel{\infty}{\Sigma}^{\infty} \frac{(\alpha ; n)(\beta ; n)}{n !(\gamma ; n) 4^{n}} F\left(\begin{array}{l}
1-n, n, 1-\gamma-n ; 1 \\
1-\alpha-n, 1-\beta-n
\end{array}\right) x^{n}=(1-x)^{-1-\alpha} \sum_{n=0}^{\infty} \phi(n ; \alpha, \beta ; \gamma)\left(\frac{x}{x-1}\right)^{n} \text {. }
$$

Now, apply (5) to the hypergeometric function on the right of the identity

and it becomes

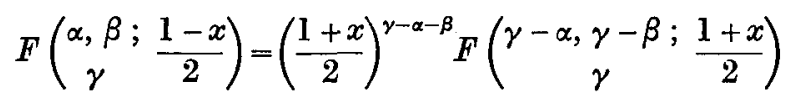

$$
\begin{aligned}
F\left(\begin{array}{c}
\alpha, \beta ; \\
\gamma
\end{array}\right. & \left.\frac{1-x}{2}\right)=\left(\frac{1+x}{2}\right)^{\gamma-\alpha-\beta} \\
& \times \sum_{=0}^{\infty} \frac{(\gamma-\alpha ; n)(\gamma-\beta ; n)}{n !(\gamma ; n) 4^{n}} F\left(\begin{array}{c}
1-n, n, 1-\gamma-n ; 1 \\
1-\gamma+\alpha-n, 1-\gamma+\beta-n
\end{array}\right)\left(1-x^{2}\right)^{n} .
\end{aligned}
$$

Next, apply (12) to the R.H.S. of (13), and so obtain

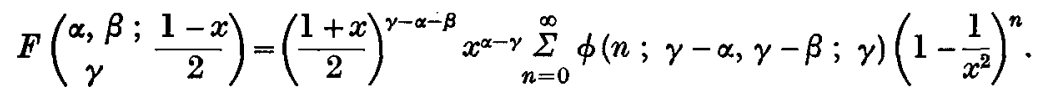

This is the first generalisation of (10), to which form it can be reduced when $\alpha+\beta=1$ by applying Whipple's formula.

The second generalisation can be derived as follows. In formula (7) replace $x$ by $1-x^{2}$ and replace $\beta$ by $\gamma-\beta$; then

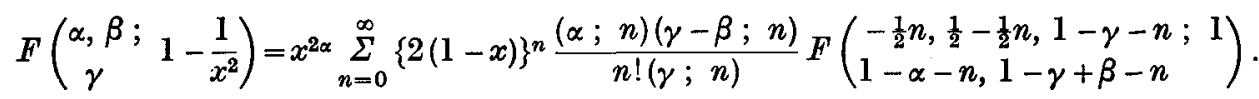

Now it can easily be verified that

$$
\begin{array}{r}
(1-x)^{2 \alpha+2 \beta-\gamma} \sum_{n=0}^{\infty}(4 x)^{n} \frac{(\alpha ; n)(\beta ; n)}{n !(\gamma ; n)} F\left(\begin{array}{c}
-\frac{1}{2} n, \frac{1}{2}-\frac{1}{2} n, 1-\gamma-n ; 1 \\
1-\alpha-n, 1-\beta-n
\end{array}\right) \\
=\sum_{n=0}^{\infty} \chi(n ; \alpha, \beta ; \gamma)(4 x)^{n},
\end{array}
$$

where

$$
\begin{aligned}
\chi(n ; \alpha, \beta ; \gamma)=\frac{(\alpha ; n)(\beta ; n)}{n !(\gamma ; n)} & \sum_{r=0}^{\infty} \frac{(\gamma-2 \alpha-2 \beta ; r)(-n ; r)(1-\gamma-n ; r)}{r !(1-\alpha-n ; r)(1-\beta-n ; r)} \cdot \frac{1}{4^{r}} \\
& \times F\left(\begin{array}{c}
\frac{r-n}{2}, \frac{1+r-n}{2}, 1-\gamma+r-n ; 1 \\
1-\alpha+r-n, 1-\beta+r-n
\end{array}\right) \cdot \ldots .
\end{aligned}
$$


Here replace $x$ by $\frac{1}{2}(1-x)$ and $\beta$ by $\gamma-\beta$ and substitute on the right of (15); then

$$
\sum_{n=0}^{\infty} x(n ; \alpha, \gamma-\beta ; \gamma)\left(4 \frac{1-x}{2}\right)^{n}=\left(\frac{1+x}{2 x}\right)^{2 \alpha-2 \beta+\gamma} x^{\gamma-2 \beta} F\left(\begin{array}{c}
\alpha, \beta ; \\
\gamma
\end{array} 1-\frac{1}{x^{2}}\right) \text {. }
$$

When $\gamma=\alpha+\beta+\frac{1}{2}$, formula (16a) can be simplified by applying Saalschutz's theorem. Formula (10) is thus obtained as a particular case.

UnIVERSity of GLasgow 